Real Analysis Exchange

Vol. 29(1), 2003/2004, pp. 947-962

Franciszek Prus-Wiśniowski and Grzegorz Szkibiel, Instytut Matematyki, Uniwersytet Szczeciński, Wielkopolska 15, 70-392 Szczecin, Poland. e-mail:

wisniows@univ.szczecin.pl and szkibiel@euler.mat.univ.szczecin.pl

\title{
SAC PROPERTY AND APPROXIMATE SEMICONTINUITY
}

\begin{abstract}
In this article we investigate approximate semicontinuity of a function related to Grande's SAC problem.
\end{abstract}

The notion of the property SAC has been introduced by Z. Grande in [2] in connection with his investigation of equiderivatives and approximate equicontinuity. A function $f: \mathbb{R} \rightarrow \mathbb{R}$ is said to have property $\mathrm{SAC}$ if for every $\eta>0$ there is an approximately continuous positive function $r: \mathbb{R} \rightarrow \mathbb{R}$ such that for every $x$ and every $h$ with $0<|h|<r(x)$ we have

$$
\left|\frac{1}{h} \int_{x}^{x+h} f(t) d t-f(x)\right|<\eta .
$$

Grande has proven that every function with property SAC must be approximately continuous. He also asked whether the converse holds, that is, whether every approximately continuous function has property SAC.

In an incomplete discussion of the above problem contained in [6] a function $p(x)$ appeared in a natural way. Below we redefine it in a slightly altered manner in order to avoid infinite values. Given an $\epsilon>0$, let

$p_{\epsilon}(x)=\sup \left\{\gamma \in(0,1]: \forall h \neq 0|h|<\gamma \Rightarrow\left|\frac{1}{h} \int_{x}^{x+h} f(t) d t-f(x)\right|<\epsilon\right\}$.

Our first lemma is devoted to an elementary inequality that will be of frequent use from us in the course of the proof of the main result of this paper.

Key Words: approximate semicontinuity, approximate continuity

Mathematical Reviews subject classification: 26A15

Received by the editors February 2, 2001 
Lemma 1 (see Lemma in [5]). Let $\sum_{i} a_{i}$ and $\sum_{i} b_{i}$ be convergent series, the first with nonnegative terms, the second one with positive terms. Then for any $k=2,3, \ldots, \infty$ the following inequalities hold

$$
\inf _{1 \leq i \leq k} \frac{a_{i}}{b_{i}} \leq \frac{\sum_{i=1}^{k} a_{i}}{\sum_{i=1}^{k} b_{i}} \leq \sup _{1 \leq i \leq k} \frac{a_{i}}{b_{i}}
$$

We will need one more auxiliary inequality that is less obvious, and we will divide its elementary but lengthy proof into two steps.

Lemma 2. Let a set $A \subset \mathbb{R}$ be union of finitely many pairwise disjoint open intervals and let $\kappa$ be any number from the interval $(0,1)$. Then for a set $B$ defined by

$$
B=\left\{y \in \mathbb{R}: \quad \exists h \neq 0 \frac{\mu([y, y+h] \cap A)}{|h|}>\kappa\right\}
$$

the following inequality holds

$$
\mu B \leq\left(\frac{2}{\kappa}-1\right) \mu A
$$

Proof. It is obvious that $A \subset B$. Further, since $\mu([y, y+h] \cap A) /|h|$ is a continuous function in $h \neq 0$, the set $B$ is open. Let us write down the set $A$ as a union of disjoint open intervals

$$
A=\bigsqcup_{i=1}^{n}\left(\alpha_{i}, \beta_{i}\right) \quad \text { with } \beta_{i}<\alpha_{i+1} \text { for } i=1, \ldots, n-1 .
$$

similarly we have

$$
B=\bigsqcup_{j=1}^{m}\left(\gamma_{j}, \delta_{j}\right) \quad \text { with } \delta_{j}<\gamma_{j+1} \text { for } j=1, \ldots, m-1 .
$$

It is not difficult to see that if $y \in B \backslash A$, then either there is an $i_{1}$ such that $\left[y, \alpha_{i_{1}}\right] \subset B$ or there is an $i_{2}$ such that $\left[\beta_{i_{2}}, y\right] \subset B$.

Next observe that it suffices to prove the inequality in the case $B$ is a single open interval. Indeed, the general case follows by setting

$$
A_{j}=A \cap\left(\gamma_{j}, \delta_{j}\right)
$$

and then using the single interval inequality

$$
\mu\left(\gamma_{j}, \delta_{j}\right) \leq\left(\frac{2}{\kappa}-1\right) \mu A_{j}
$$


and adding these inequalities for $j=1, \ldots, m$.

Assume that $B$ is a single open interval. We start with a definition of a function that will measure all holes in a set being a union of finitely many pairwise disjoint intervals. given such a set $C=\bigcup_{i=1}^{n}\left(\alpha_{i}, \beta_{i}\right)$, we put $h(C)=$ $\sup C-\inf C-\mu(C)$. We set further for any positive integer $k \leq n$

$$
A_{k}=\bigcup_{i=1}^{k}\left(\alpha_{i}, \beta_{i}\right) \quad \text { and } \quad \tilde{A}_{k}=\bigcup_{i=k}^{n}\left(\alpha_{i}, \beta_{i}\right)
$$

and define

$$
\begin{aligned}
& \gamma=\alpha_{1}-\max _{1 \leq k \leq n}\left\{\left(\frac{1}{\kappa}-1\right) \mu A_{k}-h\left(A_{k}\right)\right\} \\
& \delta=\beta_{n}+\max _{1 \leq k \leq n}\left\{\left(\frac{1}{\kappa}-1\right) \mu \tilde{A_{k}}-h\left(\tilde{A_{k}}\right)\right\} .
\end{aligned}
$$

Clearly $\gamma \leq \alpha_{1}$ and $\delta \geq \beta_{n}$. We will show that $B=(\gamma, \delta)$ in three steps.

First we will show that $x \leq \gamma$ implies $x \notin B$. Take any $x \leq \gamma$. Clearly $[x, x+h] \cap A=\varnothing$ for $h<0$. If $[x, x+h] \cap A \neq \varnothing$, then exactly one of the following cases holds

(i) $x+h \in\left(\alpha_{i}, \beta_{i}\right)$ for some $i \in\{1, \ldots, n\}$;

(ii) $x+h \in\left[\beta_{i}, \alpha_{i+1}\right]$ for some $i \in\{1, \ldots, n-1\}$;

(iii) $x+h \geq \beta_{n}$.

In the first case we get

$$
\begin{aligned}
\frac{\mu([x, x+h] \cap A)}{h} & <\frac{\left.\mu\left(\left[x, \beta_{i}\right] \cap A\right)\right]}{\beta_{i}-x}=\frac{\mu A_{i}}{\mu A_{i}+h\left(A_{i}\right)+\alpha_{1}-x} \\
& \leq \frac{\mu A_{i}}{\mu A_{i}+h\left(A_{i}\right)+\alpha_{1}-\gamma} \\
& \leq \frac{\mu A_{i}}{\mu A_{i}+h\left(A_{i}\right)+\left(\frac{1}{\gamma}-1\right) \mu A_{i}-h\left(A_{i}\right)}=\kappa .
\end{aligned}
$$

In the case (ii) we get in an analogous manner

$$
\frac{\mu([x, x+h] \cap A)}{h} \leq \frac{\mu\left(\left[x, \beta_{i}\right] \cap A\right)}{\beta_{i}-x} \leq \kappa .
$$


Similarly in the case (iii) we get

$$
\frac{\mu([x, x+h] \cap A)}{h}=\frac{\mu\left(\left[x, \beta_{n}\right] \cap A\right)}{h} \leq \frac{\mu\left(\left[x, \beta_{n}\right] \cap A\right)}{\beta_{n}-x} \leq \kappa .
$$

Thus, $x \notin B$.

The second step is devoted to proving that $x \geq \delta$ implies $x \notin B$, but we will leave it out for it is essentially the same as the first step.

In the final step we are going to show that $\gamma<x<\beta$ implies $x \in B$.

Since according to our earlier assumption $B$ has only one component, $\alpha_{1}<$ $x<\beta_{n}$ implies $x \in B$. Take $x \in\left[\beta_{n}, \delta\right)$. If $x=\beta_{n}$, it is obvious that $x \in B$. If $x>\beta_{n}$, then let $k_{0}$ be such that

$$
\left.\left(\frac{1}{\kappa}-1\right) \mu\left(\tilde{A}_{k_{0}}\right)-h\left(\tilde{A}_{k_{0}}\right)=\max _{1 \leq k \leq n}\left\{\left(\frac{1}{\kappa}-1\right) \mu\left(\tilde{A}_{k}\right)\right)-h\left(\tilde{A}_{k}\right)\right\} .
$$

Putting $h=-\left(x-\alpha_{k_{0}}\right)$, we get $h<0$, and $\mu([x, x+h] \cap A)=\mu\left(\tilde{A}_{k_{0}}\right)$, and

$$
\begin{aligned}
|h| & <\delta-\alpha_{k_{0}}=\beta_{n}+\left(\frac{1}{\kappa}-1\right) \mu\left(\tilde{A}_{k_{0}}\right)-h\left(\tilde{A}_{k_{0}}\right)-\alpha_{k_{0}} \\
& =\mu\left(\tilde{A}_{k_{0}}\right)+h\left(\tilde{A}_{k_{0}}\right)+\left(\frac{1}{\kappa}-1\right) \mu\left(\tilde{A}_{k_{0}}\right)-h\left(\tilde{A}_{k_{0}}\right)=\frac{1}{\kappa} \mu\left(\tilde{A}_{k_{0}}\right) .
\end{aligned}
$$

Therefore $x \in B$ and thus $\left[\beta_{n}, \delta\right) \subset B$. In a similar way we can show that $\left(\gamma, \alpha_{n}\right] \subset B$.

We complete the proof of the Lemma 2 by showing that $\delta-\gamma \leq\left(\frac{2}{\kappa}-1\right) \mu(A)$. To do this, we assume that $k_{1}$ and $k_{2}$ are such that

$$
\left.\left(\frac{1}{\kappa}-1\right) \mu\left(A_{k_{1}}\right)-h\left(A_{k_{1}}\right)=\max _{1 \leq k \leq n}\left\{\left(\frac{1}{\kappa}-1\right) \mu\left(A_{k}\right)\right)-h\left(A_{k}\right)\right\} .
$$

and

$$
\left.\left(\frac{1}{\kappa}-1\right) \mu\left(\tilde{A}_{k_{2}}\right)-h\left(\tilde{A}_{k_{2}}\right)=\max _{1 \leq k \leq n}\left\{\left(\frac{1}{\kappa}-1\right) \mu\left(\tilde{A}_{k}\right)\right)-h\left(\tilde{A}_{k}\right)\right\} .
$$

We have

$$
\begin{aligned}
\delta-\gamma & =\beta_{n}-\alpha_{1}+\left(\frac{1}{\kappa}-1\right) \mu\left(A_{k_{1}}\right)-h\left(A_{k_{1}}\right)+\left(\frac{1}{\kappa}-1\right) \mu\left(\tilde{A}_{k_{2}}\right)-h\left(\tilde{A}_{k_{2}}\right) \\
& =\mu(A)+h(A)+\left(\frac{1}{\kappa}-1\right) \mu\left(A_{k_{1}}\right)-h\left(A_{k_{1}}\right)+\left(\frac{1}{\kappa}-1\right) \mu\left(\tilde{A}_{k_{2}}\right)-h\left(\tilde{A}_{k_{2}}\right) .
\end{aligned}
$$


If $k_{2} \leq k_{1}$, then $h\left(A_{k_{1}}\right)+h\left(\tilde{A}_{k_{2}}\right) \geq h(A)$, so $\delta-\gamma \leq\left(\frac{2}{\kappa}-1\right) \mu(A)$. Assume now $k_{1}<k_{2}$ and denote

$$
\hat{h}\left(A_{k_{2}} \backslash A_{k_{1}}\right)= \begin{cases}h\left(A_{k_{2}} \backslash A_{k_{1}}\right) & \text { if } k_{1}>1 \\ h\left(A_{k_{2}}\right) & \text { if } k_{1}=1 .\end{cases}
$$

Then

$$
\begin{aligned}
& \left(\frac{1}{\kappa}-1\right) \mu\left(A_{k_{2}}\right)-h\left(A_{k_{2}}\right)= \\
& \quad=\left(\frac{1}{\kappa}-1\right)\left(\mu\left(A_{k_{1}}\right)+\mu\left(A_{k_{2}} \backslash A_{k_{1}}\right)\right)-h\left(A_{k_{1}}\right)-\hat{h}\left(A_{k_{2}} \backslash A_{k_{1}}\right) .
\end{aligned}
$$

Using the above equality and the fact that

$$
\left(\frac{1}{\kappa}-1\right) \mu\left(A_{k_{1}}\right)-h\left(A_{k_{1}}\right) \geq\left(\frac{1}{\kappa}-1\right) \mu\left(A_{k_{2}}\right)-h\left(A_{k_{2}}\right),
$$

we obtain

$$
0 \leq\left(\frac{1}{\kappa}-1\right) \mu\left(A_{k_{2}} \backslash A_{k_{1}}\right)
$$

By (1) and (2) we get

$$
\begin{aligned}
& \delta-\gamma \leq \mu(A)+h(A)+\left(\frac{1}{\kappa}-1\right) \mu\left(\tilde{A}_{k_{2}}\right)-h\left(\tilde{A}_{k_{2}}\right) \\
& +\left(\frac{1}{\kappa}-1\right) \mu\left(A_{k_{2}}\right)-h\left(A_{k_{2}}\right)-\left(\frac{1}{\kappa}-1\right) \mu\left(A_{k_{2}} \backslash A_{k_{1}}\right)+\tilde{h}\left(A_{k_{2}} \backslash A_{k_{1}}\right) \\
& =\frac{1}{\kappa} \mu(A)+\left(\frac{1}{\kappa}-1\right)\left(\beta_{k_{2}}-\alpha_{k_{2}}\right)-\left(\frac{1}{\kappa}-1\right) \mu\left(A_{k_{2}} \backslash A_{k_{1}}\right)+\tilde{h}\left(A_{k_{2}} \backslash A_{k_{1}}\right) .
\end{aligned}
$$

Therefore by (3)

$$
\delta-\gamma \leq \frac{1}{\kappa} \mu(A)+\left(\frac{1}{\kappa}-1\right)\left(\beta_{k_{2}}-\alpha_{k_{2}}\right) \leq\left(\frac{2}{\kappa}-1\right) \mu(A)
$$

and the proof of lemma 2 is complete.

Lemma 3. Assume $\kappa \in(0,1]$. If a set $A$ is a union of finitely many pairwise disjoint open intervals and if a set $B$ is defined by

$$
B=\left\{y \in \mathbb{R}: \quad \exists h \neq 0 \quad \frac{\mu([y, y+h] \cap A)}{|h|} \geq \kappa\right\},
$$


then

$$
\mu B \leq\left(\frac{2}{\kappa}-1\right) \mu A
$$

Proof. If $\kappa=1$, then $B=\bar{A}$ and $\mu B=\mu A=\left(\frac{2}{\kappa}-1\right) \mu A$.

Suppose $\kappa<1$. Take an $x \in B$ such that

$$
x \notin C=\left\{y: \exists h \neq 0 \frac{\mu([y, y+h] \cap A)}{|h|}>\kappa\right\} .
$$

Then in particular $x \notin \bar{A}$ and there is an $h \neq 0$ such that

$$
\frac{\mu([y, y+h] \cap A)}{|h|}=\kappa .
$$

We will consider only the case $h>0$, since the other case is quite similar. Take any $\delta \in(0, h)$ such that $(x, x+\delta) \cap A=\varnothing$. Then

$$
\frac{\mu\left(\left[x+\delta, x_{h}\right] \cap A\right)}{h-\delta}=\frac{\mu\left(\left[x, x_{h}\right] \cap A\right)}{h-\delta}>\frac{\mu\left(\left[x, x_{h}\right] \cap A\right)}{h}=\kappa .
$$

Thus $x+\delta \in C$ for sufficiently small $\delta>0$ and hence $x \in \bar{C}$ implying $B \subset \bar{C}$. Thus by the previous lemma

$$
\mu B \leq \mu \bar{C}=\mu C \leq\left(\frac{2}{\kappa}-1\right) \mu A .
$$

The proof is complete.

Lemma 4. Let a set $A \subset \mathbb{R}$ be measurable and let $\kappa \in(0,1]$. Then for a set $B$ defined by

$$
B=\left\{y \in \mathbb{R}: \quad \exists h \neq 0 \quad \frac{\mu([y, y+h] \cap A)}{|h|} \geq \kappa\right\}
$$

the following estimate holds

$$
\mu B \leq\left(\frac{2}{\kappa}-1\right) \mu A
$$

Proof. First consider the case of $A$ being an open set. Then $A$ can be written as a countable union of pairwise disjoint open intervals $A=\bigcup_{n=1}^{\infty} P_{n}$. Then denoting

$$
B_{k}=\left\{y \in \mathbb{R}: \exists h \neq 0 \frac{\mu\left([y, y+h] \cap \bigcup_{n=1}^{k} P_{n}\right)}{|h|} \geq \kappa\right\}
$$


we get $B=\bigcup B_{k}$ and $B_{k} \subset B_{k+1}$. Therefore by Lemma 3

$$
\mu B=\lim _{k} \mu B_{k} \leq \lim _{k}\left(\frac{2}{\kappa}-1\right) \mu\left(\bigcup_{n=1}^{k} P_{n}\right)=\left(\frac{2}{\kappa}-1\right) \mu A .
$$

Now consider the case of A being a $G_{\delta}$-set. If $\mu A=+\infty$, there is nothing to prove. If $A$ is of finite measure, it can be written as a countable intersection of open sets of finite measure

$$
A=\bigcap_{n=1}^{\infty} D_{n} \quad \text { where } D_{n+1} \subset D_{n} .
$$

Setting

$$
B_{k}=\left\{y \in \mathbb{R}: \quad \exists h \neq 0 \quad \frac{\mu\left([y, y+h] \cap D_{k}\right)}{|h|} \geq \kappa\right\},
$$

we get $B \subset \bigcap_{k} B_{k}$ and by the previous step of this proof

$$
\mu B \leq \lim _{k} \mu B_{k} \leq \lim _{k}\left(\frac{2}{\kappa}-1\right) \mu D_{k}=\left(\frac{2}{\kappa}-1\right) \mu A .
$$

Finally, if $A$ is an arbitrary measurable set, there is a $G_{\delta^{-}}$superset $\tilde{A}$ of equal measure. Then the set $B$ is the same for $A$ and for $\tilde{A}$. Hence by the previous step of the proof

$$
\mu B \leq\left(\frac{2}{\kappa}-1\right) \mu \tilde{A}=\left(\frac{2}{\kappa}-1\right) \mu A
$$

which completes the proof of Lemma 4.

In [5] an example has been given of an unbounded approximately continuous function such that for a suitable $\epsilon>0$ the related function $p_{\epsilon}$ is not lower semicontinuous. Actually, this may happen even for bounded approximately continuous functions as the following example shows.

Define $f: \mathbb{R} \rightarrow \mathbb{R}$ by formula

$$
f(x)= \begin{cases}4^{2 k+1} x+1-2^{2 k+1}, & \text { if } x \in\left(\frac{1}{2^{2 k+1}}-\frac{1}{4^{2 k+1}}, \frac{1}{2^{2 k+1}}\right] \\ -4^{2 k+1} x+1+2^{2 k+1}, & \text { if } x \in\left(\frac{1}{2^{2 k+1}}, \frac{1}{2^{2 k+1}}+\frac{1}{4^{2 k+1}}\right) \\ 0 & \text { otherwise }\end{cases}
$$

where $k$ runs over all nonnegative integers. The bounded function $f$ is continuous except at 0 and it is approximately continuous everywhere. One can 
elementary compute that for $\epsilon=\frac{4}{15}$ we get $p_{\epsilon}(0)=(19+\sqrt{106}) / 60$ and that

$$
p_{\epsilon}(x)=\frac{2}{15 \cdot 4^{2 k}} \text { for } x \in\left[\frac{1}{2^{2 k+1}}-\frac{1}{4^{2 k+1}}, \frac{1}{2^{2 k+1}}+\frac{1}{4^{2 k+1}}\right] \text {. }
$$

Thus $p_{\epsilon}(0)>\liminf _{x \rightarrow 0} p_{\epsilon}(x)=0$, so $p_{\epsilon}$ is not lower semicontinuous. However, according to the next proposition the function $p_{\epsilon}$ must be approximately lower semicontinuous.

Proposition 1. Let $f$ be a bounded measurable function. If $f$ is approximately continuous at a point $x_{0}$, then for any $\epsilon>0$ the function $p_{\epsilon}$ is approximately lower semicontinuous at $x_{0}$.

Proof. Let $\sigma$ be an arbitrary positive number. Set $E=\{x: \mid f(x)-$ $\left.f\left(x_{0}\right) \mid<\sigma\right\}$. We will denote the complement of $E$ by $C E$. Fix a number $\kappa \in$ $(0,1]$ arbitrarily. It follows from the approximate continuity of the function $f$ at $x_{0}$, that there is a positive number $h_{0}$ such that

$$
\frac{\mu\left(C E \cap\left[x_{0}, x\right]\right)}{\left|x-x_{0}\right|}<\frac{\kappa}{4} \quad \text { for all } x \text { with }\left|x-x_{0}\right| \leq h_{0} .
$$

Next let us define $a_{n}=x_{0}+h_{0} / 2^{n-1}$ for all positive integers $n$. We are going to show that the set

$$
\left\{x \in\left(x_{0}, a_{2}\right): \exists 0<|h|<h_{0} \frac{\mu(C E \cap[x, x+h])}{|h|} \geq \kappa\right\}
$$

has right-hand density 0 at $x_{0}$. In a similar fashion one can show that the set

$$
\left\{x \in\left(x_{0}-\frac{h_{0}}{2}, x_{0}\right): \exists 0<|h|<h_{0} \frac{\mu(C E \cap[x, x+h])}{|h|} \geq \kappa\right\}
$$

has left-hand density 0 at $x_{0}$. These facts would imply

Claim. $x_{0}$ is a point of dispersion of the set

$$
\left\{x \in \mathbb{R}: \exists 0<|h|<\frac{h_{0}}{2} \frac{\mu(C E \cap[x, x+h])}{|h|} \geq \kappa\right\} .
$$

The set

$$
A=\left\{x \in\left(x_{0}, x_{0}+\frac{h_{0}}{2}\right): \exists 0<|h|<\frac{h_{0}}{2} \frac{\mu(C E \cap[x, x+h])}{|h|} \geq \kappa\right\}
$$


is contained in the union of the following three sets

$$
\begin{gathered}
A_{1}=\bigcup_{n \geq 2}\left\{x \in\left[a_{n+1}, a_{n}\right]: \exists h \neq 0 \text { such that } x+h \in\left[a_{n+1}, a_{n}\right]\right. \\
\text { and } \left.\frac{\mu(C E \cap[x, x+h])}{|h|} \geq \kappa\right\}, \\
A_{2}=\bigcup_{n \geq 2}\left\{x \in\left[a_{n+1}, a_{n}\right] \backslash A_{1}: \exists h>0 \text { such that } x+h \in\left[a_{n}, a_{n-1}\right]\right. \\
\left.\quad \text { and } \frac{\mu(C E \cap[x, x+h])}{|h|} \geq \kappa\right\}, \\
A_{3}=\bigcup_{n \geq 2}\left\{x \in\left[a_{n+1}, a_{n}\right] \backslash A_{1}: \exists h<0 \text { such that } x+h \in\left[a_{n+2}, a_{n+1}\right]\right. \\
\text { and } \left.\frac{\mu(C E \cap[x, x+h])}{|h|} \geq \kappa\right\} .
\end{gathered}
$$

The inclusion $A \subset A_{1} \cup A_{2} \cup A_{3}$ is not obvious, since it seems possible that the inequality

$$
\frac{\mu(C E \cap[x, y])}{|x-y|} \geq \kappa
$$

may occur for some $x \in\left[a_{n+1}, a_{n}\right]$ and $y \in\left[a_{k+1}, a_{k}\right]$ with $n \geq k+2$ or for some $x \in\left[a_{n+1}, a_{n}\right], y<x_{0}$ with $|y-x|<h_{0} / 2$.

We will show that none of the two cases holds.

Given any $x \in\left[a_{n+1}, a_{n}\right], y \in\left[a_{k+1}, a_{k}\right]$ with $n \geq k+2$ we have

$$
\begin{aligned}
& \frac{\mu(C E \cap[x, y])}{|x-y|} \leq \\
& \leq \frac{\mu\left(C E \cap\left[a_{n+1}, a_{n}\right]\right)+\sum_{i=k+1}^{n-1} \mu\left(C E \cap\left[a_{i+1}, a_{i}\right]\right)+\mu\left(C E \cap\left[a_{k+1}, a_{k}\right]\right)}{\sum_{i=k+1}^{n-1}\left(a_{i}-a_{i+1}\right)}
\end{aligned}
$$


Thus by Lemma 1 we get

$$
\begin{aligned}
& \frac{\mu(C E \cap[x, y])}{|x-y|} \leq \\
\leq & \frac{\mu\left(C E \cap\left[a_{n+1}, a_{n}\right]\right)}{a_{k+1}-a_{k+2}}+\max _{k+1 \leq i \leq n-1} \frac{\mu\left(C E \cap\left[a_{i+1}, a_{i}\right]\right)}{a_{i}-a_{i+1}}+\frac{\mu\left(C E \cap\left[a_{k+1}, a_{k}\right]\right)}{a_{k+1}-a_{k+2}} .
\end{aligned}
$$

Hence by (4) and by the definition of $a_{i}$

$$
\begin{aligned}
\frac{\mu(C E \cap[x, y])}{|x-y|} & \leq \frac{\kappa}{4}+\frac{\frac{\kappa}{4}\left(a_{n}-a_{n+1}\right)}{a_{k+1}-a_{k+2}}+\frac{\frac{\kappa}{4}\left(a_{k}-a_{k+1}\right)}{\frac{a_{k}-a_{k+1}}{2}} \\
& \leq \frac{\kappa}{4}+\frac{\kappa}{4} \cdot \frac{1}{2}+\frac{\kappa}{2}<\kappa,
\end{aligned}
$$

which eliminates the first case.

If $y<x_{0}$ and $x \in\left[a_{n+1}, a_{n}\right]$ with $x-y<\frac{h_{0}}{2}$, than by Lemma 1 and by

$$
\frac{\mu(C E \cap[x, y])}{x-y} \leq \max \left\{\frac{\mu\left(C E \cap\left[y, x_{0}\right]\right)}{x_{0}-y}, \frac{\mu\left(C E \cap\left[x_{0}, x\right]\right)}{x-x_{0}}\right\} \leq \frac{\kappa}{4} .
$$

which eliminates the second case and completes the proof of the inclusion $A \subset A_{1} \cup A_{2} \cup A_{3}$. Thus in order to prove that the right-hand density $d^{+}\left(A, x_{0}\right)$ of the set $A$ at $x_{0}$ is 0 , it suffices to prove that $d^{+}\left(A_{j}, x_{0}\right)=0$ for each $j=1,2,3$.

Define

$$
\lambda_{i}=\max _{k \geq i} \frac{\mu\left(C E \cap\left[a_{k+1}, a_{k} i\right]\right)}{a_{k}-a_{k+1}} .
$$

Since $f$ is approximately continuous at $x_{0}$, the point $x_{0}$ is a dispersion point of the set $C E$ and therefore $\lambda_{i} \rightarrow 0$ as $i \rightarrow+\infty$. By Lemma 4 we get

$$
\mu A_{1} \leq\left(\frac{2}{\kappa}-1\right) \mu\left(C E \cap\left[a_{i+1}, a_{i}\right]\right) \leq\left(\frac{2}{\kappa}-1\right) \lambda_{i} \frac{h_{0}}{2^{i}} .
$$

Thus, given any $\epsilon>0$,

$$
\frac{\mu\left(A_{1} \cap\left[a_{i+1}, a_{i}\right]\right)}{a_{i}-a_{i+1}}<\epsilon
$$

for all sufficiently large $i$. Hence for those $n$ by Lemma 1

$$
\begin{aligned}
\frac{\mu\left(A_{1} \cap\left[x_{0}, a_{n}\right]\right)}{a_{n}-x_{0}} & =\lim _{k \rightarrow+\infty} \frac{\mu\left(A_{1} \cap\left[a_{n+k}, a_{n}\right]\right)}{a_{n}-a_{n+k}} \\
& \leq \liminf _{k \rightarrow+\infty} \max _{n \leq i \leq n+k-1} \frac{\mu\left(A_{1} \cap\left[a_{i+1}, a_{i}\right]\right)}{a_{i}-a_{i+1}} \leq \epsilon .
\end{aligned}
$$


Thus for any $x \in\left[a_{n+1}, a_{n}\right]$ and for those sufficiently large $n$

$$
\frac{\mu\left(A_{1} \cap\left[x_{0}, x\right]\right)}{x-x_{0}} \leq \frac{\mu\left(A_{1} \cap\left[x_{0}, a_{n}\right]\right)}{a_{n+1}-x_{0}}=2 \frac{\mu\left(A_{1} \cap\left[x_{0}, a_{n}\right]\right)}{a_{n}-x_{0}} \leq 2 \epsilon
$$

implying $d^{+}\left(A_{1}, x_{0}\right) \leq 2 \epsilon$. Since $\epsilon$ was arbitrary, we have proven that $x_{0}$ is a right-hand dispersion point of the set $A_{1}$.

Our next step is to compute the right-hand density of $A_{2}$ at $x_{0}$. Given $x \in\left[a_{n+1}, a_{n}\right], n>1$, and $h \in\left(0, h_{0} / 2\right)$ such that $x+h \in\left[a_{n}, a_{n-1}\right]$ and

$$
\frac{\mu([x, x+h] \cap C E)}{h} \geq \kappa
$$

we get

$\mu([x, x+h] \cap C E) \leq \lambda_{n}\left(a_{n}-a_{n+1}\right)+\lambda_{n-1}\left(a_{n-1}-a_{n}\right) \leq \lambda_{n-1}\left(a_{n-1}-a_{n+1}\right)$.

Hence by (5)

$$
h \leq \frac{1}{\kappa} \lambda_{n-1}\left(a_{n-1}-a_{n+1}\right)
$$

and therefore

$$
a_{n}-\frac{1}{\kappa} \lambda_{n-1}\left(a_{n-1}-a_{n+1}\right) \leq x \leq a_{n} .
$$

It follows that

$$
\frac{\left(A_{2} \cap\left[a_{n+1}, a_{n}\right]\right)}{a_{n}-a_{n+1}} \leq \frac{\lambda_{n-1}\left(a_{n-1}-a_{n+1}\right)}{a_{n+1}-a_{n+2}}=\frac{6}{\kappa} \lambda_{n-1},
$$

and thus for $x \in\left[a_{n+1}, a_{n}\right]$ by Lemma 1

$$
\begin{aligned}
\frac{\mu\left(A_{2} \cap\left[x_{0}, x\right]\right)}{x-x_{0}} & \leq \frac{\sum_{k=n}^{\infty} \mu\left(A_{2} \cap\left[a_{k+1}, a_{k}\right]\right)}{\sum_{k=n+1}^{\infty}\left(a_{k}-a_{k+1}\right)} \\
& \leq \max _{k \geq n} \frac{\mu\left(A_{2} \cap\left[a_{k+1}, a_{k}\right]\right)}{a_{k+1}-a_{k+2}} \leq \frac{6}{\kappa} \lambda_{n-1} .
\end{aligned}
$$

The last expression tends to 0 as $n$ increases. Hence $d^{+}\left(A_{2}, x_{0}\right)=0$.

The computation of the right-hand density of $A_{3}$ at $x_{0}$ is quite similar, but we will write it down for the sake of completeness. Given an $x \in\left[a_{n+1}, a_{n}\right]$, and an $h \in\left(-h_{0} / 2,0\right)$ such that $x+h \in\left[a_{n+2}, a_{n+1}\right]$ and

$$
\frac{\mu([x, x+h] \cap C E)}{h} \geq \kappa
$$


we get

$\mu([x+h, x] \cap C E) \leq \lambda_{n}\left(a_{n}-a_{n+1}\right)+\lambda_{n+1}\left(a_{n+1}-a_{n+2}\right) \leq \lambda_{n}\left(a_{n}-a_{n+2}\right)$.

Hence by (6)

$$
|h| \leq \frac{1}{\kappa} \lambda_{n}\left(a_{n}-a_{n+2}\right)
$$

and therefore

$$
a_{n+1} \leq x \leq a_{n+1}+\frac{1}{\kappa} \lambda_{n}\left(a_{n}-a_{n+2}\right)
$$

It follows that

$$
\frac{\left(A_{3} \cap\left[a_{n+1}, a_{n}\right]\right)}{a_{n+1}-a_{n+2}} \leq \frac{\lambda_{n}\left(a_{n}-a_{n+2}\right)}{a_{n+1}-a_{n+2}}=\frac{3}{\kappa} \lambda_{n},
$$

and thus for $x \in\left[a_{n+1}, a_{n}\right]$ by Lemma 1

$$
\begin{aligned}
\frac{\mu\left(A_{3} \cap\left[x_{0}, x\right]\right)}{x-x_{0}} & \leq \frac{\sum_{k=n}^{\infty} \mu\left(A_{3} \cap\left[a_{k+1}, a_{k}\right]\right)}{\sum_{k=n+1}^{\infty}\left(a_{k}-a_{k+1}\right)} \\
& \leq \max _{k \geq n} \frac{\mu\left(A_{3} \cap\left[a_{k+1}, a_{k}\right]\right)}{\left.a_{k+1}-a_{k+2}\right)} \leq \frac{3}{\kappa} \lambda_{n-1} .
\end{aligned}
$$

The last expression tends to 0 as $n$ increases. Hence $d^{+}\left(A_{2}, x_{0}\right)=0$. Therefore $d^{+}\left(A, x_{0}\right)=0$ which completes the proof of our claim.

We now return to our investigation of the behavior of the function $p_{\epsilon}$. Let $f$ be approximately continuous at $x_{0}$ and let $M>0$ be a constant such that $|f(x)| \leq M / 2$ for all $x \in \mathbb{R}$. Fix $\epsilon>0$ arbitrarily. Denote the set $\left\{x:\left|f(x)-f\left(x_{0}\right)\right|<\epsilon / 3\right\}$ by $A$. Then by our claim there is an $h_{0}>0$ such that the set

$$
B=\left\{x: \quad \forall h \neq 0 \quad|h|<\frac{h_{0}}{2} \Rightarrow \frac{\mu(C A \cap[x, x+h])}{|h|}<\frac{\epsilon}{3 M}\right\}
$$

has density one at $x_{0}$. Approximate continuity of $f$ at $x_{0}$ implies that $d\left(A, x_{0}\right)=$ 1 and therefore $d\left(A \cap B, x_{0}\right)=1$. We will show that $p_{\epsilon}(x) \geq h_{0} / 2$ for 
$x \in A \cap B$. Take any $x \in A \cap B$ and take any $h$ with $|h| \in\left(0, \frac{h_{0}}{2}\right)$. Then

$$
\begin{aligned}
& \left|\frac{1}{h} \int_{x}^{x+h} f(t) d t-f(x)\right|=\left|\frac{1}{h} \int_{x}^{x+h}(f(t)-f(x)) d t\right| \\
& \quad \leq \frac{1}{|h|} \int_{[x, x+h]}\left(\left|f(t)-f\left(x_{0}\right)\right|+\left|f\left(x_{0}\right)-f(x)\right|\right) d t \\
& \quad<\frac{1}{|h|} \int_{[x, x+h] \cap A}\left|f(t)-f\left(x_{0}\right)\right| d t+\frac{1}{|h|} \int_{[x, x+h] \cap C A}\left|f(t)-f\left(x_{0}\right)\right| d t+\frac{\epsilon}{3} \\
& \leq \frac{\epsilon}{3}+\frac{1}{|h|} \cdot \frac{\epsilon|h|}{3 M} \cdot M+\frac{\epsilon}{3}=\epsilon .
\end{aligned}
$$

Thus $p_{\epsilon}(x) \geq h_{0} / 2$.

Take an arbitrary $\gamma>0$. If $h_{0} / 2 \geq p_{\epsilon}(x)-\gamma$, then

$$
\operatorname{app} \liminf _{x \rightarrow x_{0}} p_{\epsilon}(x) \geq \liminf _{A \cap B \ni x \rightarrow x_{0}} p_{\epsilon}(x) \geq \frac{h_{0}}{2} \geq p_{\epsilon}(x)-\gamma
$$

Otherwise, since $\left|\frac{1}{h} \int_{x_{0}}^{x_{0}+h} f(t) d t-f\left(x_{0}\right)\right|$ is a continuous function in $h$, it attains its maximum on a set $Z=\left\{h: h_{0} / 2 \leq|h| \leq p_{\epsilon}\left(x_{0}\right)-\gamma\right\}$ and hence we get

$$
\inf _{h \in Z}\left\{\epsilon\left|\frac{1}{h} \int_{x_{0}}^{x_{0}+h} f(t) d t-f\left(x_{0}\right)\right|\right\}>0
$$

denote half of the minimum by $\eta$ and set $E=\left\{x:\left|f(x)-f\left(x_{0}\right)\right|<\eta / 2\right\}$. Take any $x \in E \cap A \cap B$ such that

$$
\frac{4\left|x-x_{0}\right| M}{h_{0}}<\frac{\eta}{2}
$$


and take any $h \in Z$. Then

$$
\begin{aligned}
|| f(x)-\frac{1}{h} \int_{x}^{x+h} f(t) d t|-| f\left(x_{0}\right)-\frac{1}{h} \int_{x_{0}}^{x_{0}+h} f(t) d t|| \\
\quad \leq\left|f(x)-f\left(x_{0}\right)\right|+\left|\frac{1}{h} \int_{x}^{x+h} f(t) d t-\frac{1}{h} \int_{x_{0}}^{x_{0}+h} f(t) d t\right| \\
\leq \frac{\eta}{2}+\frac{2\left|x-x_{0}\right| M}{h} \leq \frac{\eta}{2}+\frac{4\left|x-x_{0}\right| M}{h_{0}}<\eta .
\end{aligned}
$$

Thus

$$
\left|f(x)-\frac{1}{h} \int_{x}^{x+h} f(t) d t\right| \leq\left|f\left(x_{0}\right)-\frac{1}{h} \int_{x_{0}}^{x_{0}+h} f(t) d t\right|+\eta<\epsilon,
$$

where the latter inequality is valid by (7). Since $x \in A \cap B$, we get

$$
\left|f(x)-\frac{1}{h} \int_{x}^{x+h} f(t) d t\right|<\epsilon
$$

for all $h \neq 0$ such that $|h| \leq p_{\epsilon}\left(x_{0}\right)-\gamma$. Thus

$$
p_{\epsilon}(x) \geq p_{\epsilon}\left(x_{0}\right)-\gamma
$$

for $x \in E \cap A \cap B$. Therefore

$$
\text { app } \liminf _{x \rightarrow x_{0}} p_{\epsilon}(x) \geq p_{\epsilon}\left(x_{0}\right)-\gamma .
$$

Since $\gamma$ was arbitrary, it completes the proof of approximate lower semicontinuity of $p_{\epsilon}$ at the point $x_{0}$.

Proposition 2. Let $f$ be bounded and measurable. If $f$ is approximately continuous at a point $x_{0}$, then for all $\epsilon>0$ the function

$$
\bar{p}_{\epsilon}(x)=\sup \left\{\gamma \in(0,1]: \forall h \neq 0|h|<\gamma \Rightarrow\left|\frac{1}{h} \int_{x}^{x+h} f(t) d t-f(x)\right| \leq \epsilon\right\}
$$

is approximately upper semicontinuous at $x_{0}$. 
Proof. If $\lim \sup _{x \rightarrow x_{0}} \bar{p}_{\epsilon}(x)=0$, then $\bar{p}_{\epsilon}$ is upper semicontinuous at $x_{0}$ for it takes positive values everywhere.

Assume now that $\limsup _{x \rightarrow x_{0}} \bar{p}_{\epsilon}(x)=\delta>0$ and take any $\gamma \in(0, \delta)$. Then the set $E_{1}=\left\{x: \bar{p}_{\epsilon}(x)>\gamma\right\}$ has positive upper density at $x_{0}$. Let $E_{2}$ be a set of density one at $x_{0}$ such that $\left.f\right|_{E_{2}}$ is continuous. Then there is a sequence $\left(x_{n}\right)$ of points of $E_{1} \cap E_{2}$ convergent to $x_{0}$ such that $x_{n} \neq x_{0}$ for all $n$. It is easy to see that for all $0<|h| \leq \gamma$ and for all positive integers $n$

$$
\left|\int_{x_{n}}^{x_{n}+h} f(t) d t-f\left(x_{n}\right)\right| \leq \epsilon .
$$

Passing to a limit with $n \rightarrow \infty$, we get

$$
\left|\int_{x_{0}}^{x_{0}+h} f(t) d t-f\left(x_{0}\right)\right| \leq \epsilon .
$$

Therefore $\bar{p}_{\epsilon}\left(x_{0}\right) \geq \gamma$. Since $\gamma<\delta$ was arbitrary, we have $\bar{p}_{\epsilon}\left(x_{0}\right) \geq \delta$ and the proof is complete.

The SAC problem would be solved if it were possible to construct an approximately continuous function $r$ such that $0<r \leq p_{\epsilon}$. In [6] we have shown that if $f$ is a positive approximately lower semicontinuous function of Baire class one then the required function $r$ can be found. However, there is no guarantee that our function $p_{\epsilon}$ is Baire class one and this assumption is crucial as the following example shows.

Let $\left(K_{n}\right)_{n \in \mathbb{N}}$ be a partition of all rationals from the interval $[0,1]$ into countably many subsets each of which is dense in the whole interval. Define $f$ to be $\frac{1}{n+1}$ on $K_{n}$ and to be 1 on the irrationals. It is obvious that $f$ is approximately lower semicontinuous and that it is not of Baire class one. It is easy to see that there is no approximately continuous function $r$ such that $0<r \leq f$. Since each of the sets $K_{n}$ is dense in [0,1], so are the sets where $r$ is less than any given positive value, and hence every point of the interval is a point of discontinuity of $r$. This can not happen for $r$ is of Baire class one.

Open Problem Find a characterization of those approximately lower semicontinuous functions $f>0$ for which there is an approximately continuous function $r$ such that $0<r \leq f$.

\section{References}

[1] A.Bruckner, Differentiation of Real Functions, CRM Monograph Series, American Mathematical Society, Providence 1994 
[2] Z. Grande, On Equi-derivatives, Real Analysis Exchange 21(1995/96) 637647

[3] R. Kannan, C. King Krueger, Advanced Analysis on the Real Line, Springer Verlag, New York 1996

[4] S. Eojasiewicz, An Introduction to the Theory of Real Functions, John Wiley\&Sons, Chichester 1988

[5] F. Prus-Wiśniowski, SAC Property and semicontinuity, to appear in Tatra Mountains Math. Publ.

[6] F. Prus-Wiśniowski, G. Szkibiel, A Note on SAC Property, Tatra Mountains Math. Publ., 19(2000) 209-212 